\title{
Expression of PDK-1 and DMBT1 in the thyroid carcinoma and its clinicopathological significance
}

\author{
ZHICHAO SUN and LEI XU \\ Department of Pathology, The Second People's Hospital of Lianyungang, \\ Lianyungang, Jiangsu 222006, P.R. China
}

Received November 6, 2018; Accepted May 10, 2019

DOI: $10.3892 /$ ol.2019.10639

\begin{abstract}
This study was designed to explore the expression of phosphoinositide-dependent protein kinase 1 (PDK-1), deleted in malignant brain tumors (DMBT1) in the thyroid carcinoma. A total of 87 fresh samples of thyroid carcinoma from surgical resection in The Second People's Hospital of Lianyungang from June 2016 to March 2018 were collected for the ELISA to detect the protein expression of PDK-1 and DMBT1. Then the pathological significance of the expression of PDK-1 and DMBT1 in the thyroid carcinoma and the correlation between them were analyzed, using the ROC curve to study the diagnostic value of each index. The expression of PDK-1 in the thyroid carcinoma tissue was significantly higher than that in the normal thyroid tissue with a statistical difference between them $(\mathrm{P}<0.05)$; the expression of DMBT1 in the thyroid carcinoma was statistically significantly lower than that in the normal thyroid tissue $(\mathrm{P}<0.05)$; the PDK-1 and DMBT1 expressions were in negative correlation in the thyroid carcinoma $(r=-0.889, \mathrm{P}<0.001)$. The AUG, specificity and the sensitivity of the PDK-1 in diagnosing the thyroid carcinoma were $0.862,86.21 \%$ and $78.16 \%$, respectively; the AUG, specificity and the sensitivity of the DMBT1 in diagnosing the thyroid carcinoma were $0.708,66.67 \%$ and $67.82 \%$, respectively; while the AUG, the specificity and the sensitivity of the combination of PDK-1 and DMBT1 in diagnosing the thyroid carcinoma were $0.888,89.66 \%$ and $81.61 \%$. In conclusion, the occurrence and progression of the thyroid carcinoma were related to the high expression of the PDK-1 and the low expression of the DMBT1 in the thyroid carcinoma tissues, the two of which were in connection with factors involving lymph node metastasis, pathological type, neoplasm staging, and clinical staging. Thus, the combined detection of PDK-1
\end{abstract}

Correspondence to: Dr Zhichao Sun, Department of Pathology, The Second People's Hospital of Lianyungang, 41 Hailian East Road, Haizhou, Lianyungang, Jiangsu 222006, P.R. China

E-mail: cdb7ta@163.com

Key words: thyroid carcinoma, phosphoinositide-dependent protein kinase 1 , deleted in malignant brain tumors, case parameters and DMBT1 could be used as an effective index to determine the occurrence of thyroid carcinoma.

\section{Introduction}

Thyroid carcinoma, the most common endocrine carcinoma (1), is seeing an increasingly high incidence worldwide in recent years (2), with a $10^{\text {th }}$-ranking incidence among tumors in China (3). Recently more scholars have a rising interest in the pathogenesis of thyroid carcinoma. Phosphoinositide-dependent protein kinase 1 (PDK-1), a monomeric polypeptide enzyme consisting of more than five hundred amino acids including the $\mathrm{N}$-terminal kinase domain and the C-terminal $\mathrm{PH}$ domain, which is normally expressed in a variety of peripheral tissues including the heart, stomach, spleen, kidney, brain and other tissues, has a ligand that plays an important role in the regulation of the immune system, tumor metastasis, embryonic development and angiogenesis (4). Studies showed that the expression level of deleted in malignant brain tumors (DMBT1) was greatly decreased in breast carcinoma (5), human oral squamous cell carcinoma (6) compared with that in the normal tissues, which suggested the potential ability of DMBT1 gene as a tumor suppressor gene to get involved in the regulation of the occurrence and development of the carcinogenesis of tumor cells. In recent years, few studies of the expression levels of DMBT and PDK-1 gene in the thyroid tissue have been reported. In the following parts, this study investigated the expression levels of PDK-1 and DMBT1 genes in the thyroid tissue and the correlation between the two, and further explored the relationship between the expression levels of PDK-1 and DMBT1 and the molecular biological mechanisms of the occurrence, development, metastasis, proliferation and malignant transformation of the thyroid carcinoma.

\section{Patients and methods}

General information. Eighty-seven patients (34 males and 53 females) with thyroid carcinoma diagnosed in The Second People's Hospital of Lianyungang (Lianyungang, China) from June 2016 to March 2018 were selected as the research subjects. Inclusion criteria: i) adults with no history of mental illness who were capable of normal communication, self-care, and clear consciousness and ii) patients (or their immediate 
Table I. Basic information of patients.

\begin{tabular}{lc}
\hline Factors & $\mathrm{n}=87$ \\
\hline Age (year) & \\
$<35$ & $31(35.63)$ \\
$\geq 35$ & $56(64.37)$ \\
Sex & \\
Male & $52(59.77)$ \\
Female & $35(40.23)$ \\
Obesity & \\
Yes & $47(54.02)$ \\
No & $40(45.98)$ \\
Clinical staging & \\
Stage I-II & $31(35.63)$ \\
Stage III-IV & $56(64.37)$ \\
Pathological type & \\
Undifferentiated & $51(58.62)$ \\
Differentiated & $36(41.38)$ \\
Size of tumor & \\
Microcarcinoma & $52(59.77)$ \\
Non-small cell carcinoma & $35(40.23)$ \\
\hline
\end{tabular}

families) that signed the informed consent. Exclusion criteria: i) patients with permanent intracardiac conduction disorder, cardiogenic shock and a history of severe mental illness and ii) patients with acute inflammation, hematological disease, and severe heart and liver dysfunction. Patients in this study received no chemotherapy, radiotherapy or immunotherapy ever. Immediately after the surgery, the resected thyroid carcinoma tissues and normal tissues over $2 \mathrm{~cm}$ away from the carcinoma with no observed cancer cell infiltration were stored in the refrigerator at $-80^{\circ} \mathrm{C}$ (Table I).

The study was approved by the Ethics Committee of The Second People's Hospital of Lianyungang. Patients who participated in this research had complete clinical data. The signed informed consents were obtained from the patients or the guardians.

Main reagents and instruments. DMBT1 ELISA test kit for human (article no. IC-DMBT1-Hu; Shanghai Yu Bo Biotechnology Co., Ltd., Shanghai, China), PDK-1 ELISA test kit for human (article no. EH15196; Wuhan Fine Biotech Co., Ltd., Wuhan, China).

Experimental methods. This experiment was carried out in a sterile environment, with all the instruments autoclaved and dried before the use. The normal thyroid tissue and the thyroid carcinoma tissue were taken out from the $-80^{\circ} \mathrm{C}$ refrigerator and thawed. Then, $0.1 \mathrm{~g}$ of normal thyroid tissue and $0.1 \mathrm{~g}$ of thyroid carcinoma were weighted. With a small amount of liquid nitrogen, the tissues were separately crushed into powder in the mortar quickly. Next, the powder was transferred into the 2 ml-sized Ep tube, afterward got mixed together with $1.2 \mathrm{ml}$ of PBS (pH 7.3) at the speed of $1,500 \mathrm{x} \mathrm{g}$ and got centrifugalized at $4^{\circ} \mathrm{C}$ for $20 \mathrm{~min}$ to get the supernatant liquid. Then the homogenate of normal

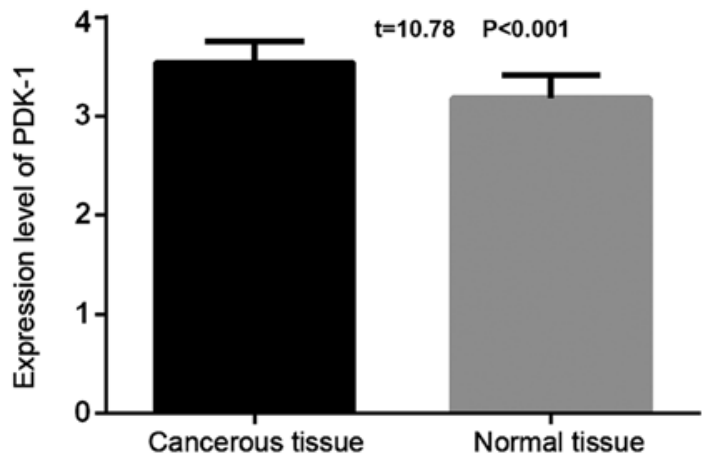

Figure 1. The expressions of PDK-1 in the thyroid carcinoma tissues and their adjacent normal tissues. The expression level of PDKP-1 in the thyroid carcinoma tissues is greatly higher than that in their adjacent normal tissues, with a statistical difference $(t=10.78, P<0.001)$. PDK-1, phosphoinositide-dependent protein kinase.

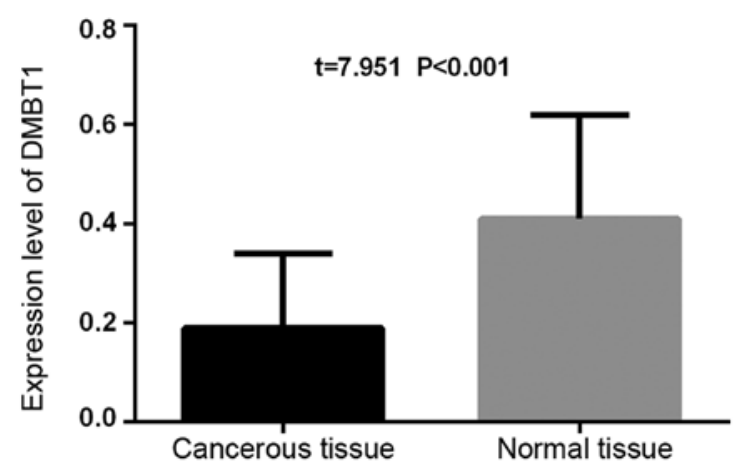

Figure 2. The expressions of DMBT1 in the thyroid carcinoma tissues and their adjacent normal tissues. The expression level of DMBT1 in the thyroid carcinoma tissues is statistically greatly lower than that in their adjacent normal tissues $(\mathrm{t}=7.95, \mathrm{P}<0.001)$. DMBT1, deleted in malignant brain tumors.

thyroid tissue and thyroid carcinoma tissue was detected in strict accordance with the instructions of the DMBT1 ELISA kit for human and the PDK-1 ELISA kit for human.

Statistical analysis. The SPSS 20.0 software (IBM Corp., Armonk, NY, USA) was used to perform the statistical analysis. The basic count data of patients were expressed as the percentage [n (\%)]; the expression levels of DMBT1 and PDK-1 were expressed as the mean \pm standard deviation (SD). Pearson's correlation was used for the correlation between the expression of PDK-1 and DMBT1. The t-test was used for statistical analysis and $\mathrm{P}<0.05$ was considered to indicate a statistically significant difference.

\section{Results}

Expression levels of DMBT1 and PDK-1 in thyroid carcinoma tissues and normal tissues adjacent to them. The expression level of PDK-1 in the thyroid carcinoma tissue $(3.54 \pm 0.21)$ was statistically higher than that of the adjacent normal thyroid tissues $(3.18 \pm 0.23 ; \mathrm{t}=10.78, \mathrm{P}<0.001)$. The expression level of PDK-1 in the thyroid carcinoma tissue $(0.19 \pm 0.15)$ was lower than that of the adjacent normal thyroid tissues $(0.41 \pm 0.21)$, and the difference was statistically significant $(\mathrm{t}=7.951, \mathrm{P}<0.001$; Figs. 1 and 2). 
Table II. Relationship between the expression level of PDK-1 and clinicopathological features of patients with thyroid carcinoma.

\begin{tabular}{|c|c|c|c|}
\hline Factors & PDK-1 & $\mathrm{t}$ & P-value \\
\hline \multicolumn{4}{|l|}{ Age (year) } \\
\hline$<35$ & $3.41 \pm 1.52$ & 1.166 & 0.245 \\
\hline$\geq 35$ & $3.67 \pm 1.42$ & & \\
\hline \multicolumn{4}{|l|}{ Gender } \\
\hline Male & $3.41 \pm 1.79$ & 0.988 & 0.325 \\
\hline Female & $3.67 \pm 1.68$ & & \\
\hline \multicolumn{4}{|l|}{ Clinical staging } \\
\hline Stages I-II & $3.21 \pm 1.47$ & 2.758 & 0.007 \\
\hline Stages III-IV & $3.87 \pm 1.68$ & & \\
\hline \multicolumn{4}{|l|}{ Lymph node metastasis } \\
\hline Yes & $3.94 \pm 1.56$ & 3.549 & $<0.001$ \\
\hline No & $3.14 \pm 1.41$ & & \\
\hline \multicolumn{4}{|l|}{ Pathological type } \\
\hline Undifferentiated & $4.56 \pm 1.71$ & 8.536 & $<0.001$ \\
\hline Differentiated & $2.52 \pm 1.43$ & & \\
\hline \multicolumn{4}{|l|}{ Size of tumors } \\
\hline Microcarcinoma & $2.30 \pm 1.32$ & 11.43 & $<0.001$ \\
\hline Non-small carcinoma & $4.88 \pm 1.64$ & & \\
\hline
\end{tabular}

PDK-1, phosphoinositide-dependent protein kinase.

Relationship between the expression levels of DMBT1 and $P D K-1$ and the clinicopathological features of patients with thyroid carcinoma

Relationship between the expression level of PDK-1 and the clinicopathological features of patients with thyroid carcinoma. The expression level of PDK-1 in the thyroid carcinoma tissues was significantly lower than that in the adjacent normal thyroid tissues, and the difference was statistically significant $(\mathrm{P}<0.001)$. The expression of PDK-1 in thyroid carcinoma was proved to be greatly correlated with factors like pathological type, clinical stage, tumor size and lymph node metastasis $(\mathrm{P}<0.001)$, in no significant correlation with factors such as sex and age $(\mathrm{P}>0.05)$. In the thyroid carcinoma tissues, the expression of PDK-1 in the lymph node metastasis $(3.94 \pm 1.56)$ was statistically greatly higher than that in the places without lymph node metastasis $(3.14 \pm 1.41$; $\mathrm{P}<0.001)$. The PDK-1 expression in the undifferentiated tissues $(4.56 \pm 1.71)$ was much higher than that in the differentiated thyroid carcinoma tissues $(2.52 \pm 1.43)$, with a statistical difference $(\mathrm{P}<0.001)$. The expression level of PDK-1 in the thyroid carcinoma tissues at stage I to stage II $(3.21 \pm 1.47)$ was statistically significantly lower than that in the thyroid carcinoma tissues at stage III to stage IV $(3.87 \pm 1.68$; $\mathrm{P}<0.05)$. The expression level of PDK-1 in the microcarcinoma tissues (2.30 \pm 1.32$)$ was greatly lower than that in the non-small carcinoma thyroid tissues $(4.88 \pm 1.64)$, and the difference was statistically significant $(\mathrm{P}<0.001$; Table II).

Relationship between the expression level of DMBTI and the clinicopathological features of patients with thyroid carcinoma. The expression level of DMBT1 in the thyroid carcinoma tissues was significantly lower than that that in the adjacent normal thyroid tissues, and the difference was statistically significant $(\mathrm{P}<0.001)$. The expression of DMBT1 in thyroid carcinoma was proved to be greatly correlated with factors like pathological type, clinical stage, tumor size and lymph node metastasis $(\mathrm{P}<0.001)$, in no significant correlation with factors such as sex and age $(\mathrm{P}>0.05)$. In the thyroid carcinoma tissues, the expression of DMBT1 in the lymph node metastasis $(0.17 \pm 0.14)$ was statistically lower than that in the places without lymph node metastasis $(0.21 \pm 0.11$; $\mathrm{P}<0.001)$. The DMBT1 expression in the differentiated tissues $(0.23 \pm 0.14)$ was much higher than that in the undifferentiated thyroid carcinoma tissues $(0.15 \pm 0.08)$, with a statistical difference $(\mathrm{P}<0.001)$. The expression level of DMBT1 in the thyroid carcinoma tissues at stage I to stage II $(0.22 \pm 0.14)$ was statistically significantly higher than that in the thyroid carcinoma tissues at stage III to stage IV $(0.16 \pm 0.09 ; \mathrm{P}<0.05)$. The expression level of DMBT1 in the microcarcinoma tissues $(0.27 \pm 0.13)$ was greatly higher than that in the non-small carcinoma thyroid tissues $(0.11 \pm 0.09)$, and the difference was statistically significant $(\mathrm{P}<0.001$; Table III).

Correlation between the expression of DMBT1 and the expression of $P D K-1$ in the thyroid carcinoma. Correlation analysis showed that the expressions of DMBT1 and PDK-1 were negatively correlated in the thyroid carcinoma $(r=-0.889$, $\mathrm{P}<0.001$ ) (Fig. 3).

Clinical diagnostic value of DMBT1 and PDK-1. According to the ROC curve, the AUG, the specificity, and the sensitivity of the protein PDK-1 in diagnosing thyroid carcinoma were $0.862,86.21 \%$ and $78.16 \%$, respectively, with the best cut-off point for diagnosing thyroid carcinoma of 199; the AUG, 
Table III. Relationship between the expression level of DMBT1 and the clinicopathological features of patients with thyroid carcinoma.

\begin{tabular}{|c|c|c|c|}
\hline Factors & DMBT1 & $\mathrm{t}$ & P-value \\
\hline \multicolumn{4}{|l|}{ Age (year) } \\
\hline$<35$ & $0.20 \pm 0.12$ & 1.244 & 0.215 \\
\hline$\geq 35$ & $0.18 \pm 0.09$ & & \\
\hline \multicolumn{4}{|l|}{ Gender } \\
\hline Male & $0.20 \pm 0.12$ & 1.146 & 0.253 \\
\hline Female & $0.18 \pm 0.11$ & & \\
\hline \multicolumn{4}{|l|}{ Clinical staging } \\
\hline Stages I-II & $0.22 \pm 0.14$ & 3.363 & 0.001 \\
\hline Stages III-IV & $0.16 \pm 0.09$ & & \\
\hline \multicolumn{4}{|l|}{ Lymph node metastasis } \\
\hline No & $0.21 \pm 0.11$ & 2.096 & 0.037 \\
\hline Yes & $0.17 \pm 0.14$ & & \\
\hline \multicolumn{4}{|l|}{ Pathological type } \\
\hline Differentiated & $0.23 \pm 0.14$ & 4.628 & $<0.001$ \\
\hline Undifferentiated & $0.15 \pm 0.08$ & & \\
\hline \multicolumn{4}{|l|}{ Size of tumors } \\
\hline Microcarcinoma & $0.27 \pm 0.13$ & 9.439 & $<0.001$ \\
\hline Non-small carcinoma & $0.11 \pm 0.09$ & & \\
\hline
\end{tabular}

DMBT1, deleted in malignant brain tumors.

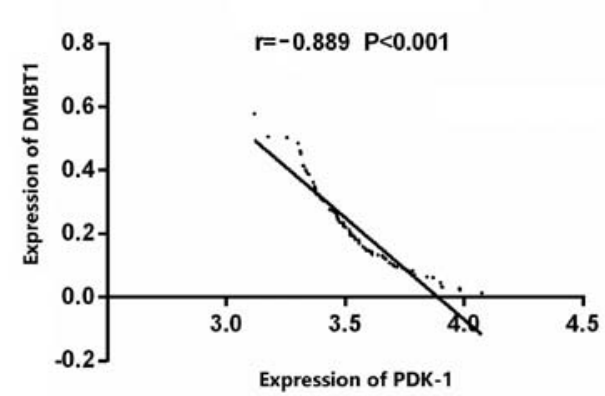

Figure 3. The correlation between the expression of PDK-1 and the expression of DMBT1 in the thyroid carcinoma. According to Pearson's correlation analysis, the expression of PDK-1 and the expression of DMBT1 are negatively related $(\mathrm{r}=-0.889, \mathrm{P}<0.001)$. PDK-1, phosphoinositide-dependent protein kinase; DMBT1, deleted in malignant brain tumors.

the specificity, and the sensitivity of the protein DMBT1 in diagnosing thyroid carcinoma were $0.708,66.67 \%$ and $67.82 \%$, respectively, with the best cut-off point for diagnosing thyroid carcinoma of 199; and the AUG, the specificity, and the sensitivity of the combination of protein PDK-1 and protein DMBT1 in diagnosing thyroid carcinoma were $0.888,89.66 \%$ and $81.61 \%$, respectively, with the best cut-off point for diagnosing thyroid carcinoma of 199 (Figs. 4, 5 and 6).

\section{Discussion}

Citing from the American Carcinoma Society, 37,340 new cases (about $0 \%$ women) of thyroid carcinoma were diagnosed in the

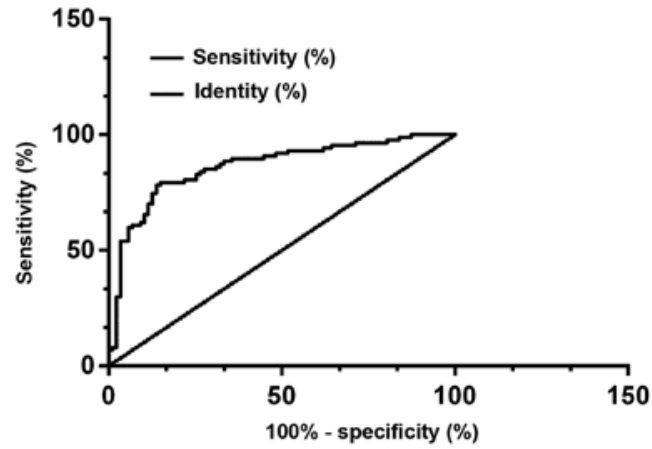

Figure 4. The diagnostic value of the expression of PDK-1 for the thyroid carcinoma. ROC was used to evaluate the diagnostic value of protein PDK-1 in thyroid cancer. PDK-1, phosphoinositide-dependent protein kinase.

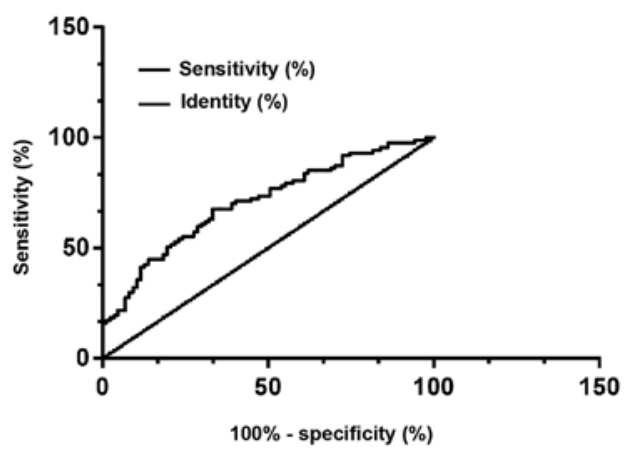

Figure 5. The diagnostic value of the expression of DMBT1 for the thyroid carcinoma. The diagnostic value of protein DMBT1 in thyroid cancer was evaluated using the ROC curve. DMBT1, deleted in malignant brain tumors. 


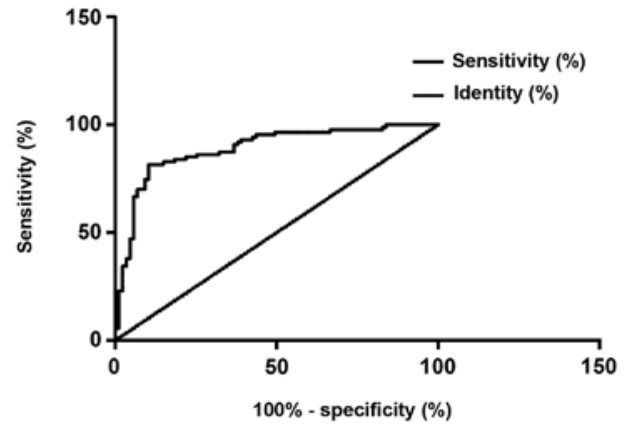

Figure 6. The diagnostic value of the combined detection the expressions of PDK-1 and DMBT1 for the thyroid carcinoma. The diagnostic value of PDK-1 combined with DMBT1 inthyroid cancer was evaluated by ROC curve. PDK-1, phosphoinositide-dependent protein kinase; DMBT1, deleted in malignant brain tumors.

United States in 2018, making the thyroid carcinoma the sixth destructive carcinoma among women (7). Thyroid carcinoma, a common malignant tumor in the head and neck (8), is prone to spread and metastasis in its early stage (9). PDK1, as the main regulator of the upstream AGG family that plays an important role in the regulation of pathological processes such as cell growth, differentiation, and apoptosis $(10,11)$, is found in recent years to be highly expressed in non-small cell carcinoma, breast carcinoma, and pancreatic carcinoma tissues (12-14). The close relation between the deterioration and the occurrence of tumors is publicly known, and the PDK1, as an important kinase in cells, has a significant regulatory effect on the cell reproduction $(15,16)$. Located on the long arm of the human's number 10 chromosome (17), the DMBT1 gene has its sequence that is mainly composed of repeated highly homologous exons and introns (18). Some studies discovered that the expression level of DMBT1 gene in tumors like esophageal carcinoma (19), colon carcinoma (20), bladder carcinoma (21), prostate carcinoma (5), and non-small cell carcinoma (22) was significantly decreased compared with its expression in normal tissues, suggesting that DMBT1 might be able to inhibit the development of oncogenes. According to the study of Mollenhauer's team (23), DMBT1 might play an important role in the development of one unclear part of the human body. It's likely that DMBT1 may inhibit the occurrence of tumors by promoting cell differentiation since the formation of tumors can lead to cell disorders. This study is the first one to detect the expression of PDK-1 and DMBT1 proteins in the thyroid carcinoma tissues and in their adjacent normal tissues more than $2 \mathrm{~cm}$ away with no carcinoma cells infiltrating using the ELISA method and analyze the correlation between the two and the pathological significance of the thyroid carcinoma. The results of this study revealed that the expression level of PDK-1 in the thyroid carcinoma tissues was statistically significantly higher than that in the normal tissues adjacent to the carcinoma $(t=10.78, P<0.001)$, while the expression level of DMBT1 in the thyroid carcinoma tissues was statistically significantly lower than that in the normal tissues adjacent to carcinoma $(\mathrm{t}=7.491, \mathrm{P}<0.05)$. Previous studies showed that the positive expression of DMBT1 in breast carcinoma was significantly lower than that in the normal tissues (24), which helpfully supports the results of this study. By now no relevant study of the expression level of PDK-1 in the thyroid carcinoma tissues has been reported. This study also analyzed the relationship between the expression levels of DMBT1 and PDK-1 and the clinicopathological features of patients with thyroid carcinoma. The results showed that the expression of PDK-1 in thyroid carcinoma was proved to be greatly correlated with factors like pathological type, clinical stage, tumor size and lymph node metastasis $(\mathrm{P}<0.001)$, in no significant correlation with factors such as sex and age $(\mathrm{P}>0.05)$; in the thyroid carcinoma tissues, the expression of PDK-1 in the lymph node metastasis was statistically greatly higher than that in the places without lymph node metastasis $(\mathrm{P}<0.001)$; the PDK-1 expression in the undifferentiated tissues was much higher than that in the differentiated thyroid carcinoma tissues, with a statistical difference $(\mathrm{P}<0.001)$; the expression level of PDK-1 in the thyroid carcinoma tissues at stage III to stage IV was statistically significantly higher than that in the thyroid carcinoma tissues at stage I to stage II $(\mathrm{P}<0.05)$; the expression level of PDK-1 in the non-small carcinoma thyroid tissues was greatly higher than that in the microcarcinoma tissues, and the difference was statistically significant $(\mathrm{P}<0.001)$. The expression of DMBT1 in the thyroid tissues was in a contrary situation: in the thyroid carcinoma tissues, the expression of DMBT1 in the lymph node metastasis was statistically greatly lower than that in the places without lymph node metastasis; the DMBT1 expression in the undifferentiated tissues was much lower than that in the differentiated thyroid carcinoma tissues, with a statistical difference $(\mathrm{P}<0.001)$; the expression level of DMBT1 in the thyroid carcinoma tissues at stage III to stage IV was statistically significantly lower than that in the thyroid carcinoma tissues at stage I to stageII $(\mathrm{P}<0.05)$; the expression level of DMBT1 in the non-small carcinoma thyroid tissues was greatly lower than that in the microcarcinoma tissues, and the difference was statistically significant $(\mathrm{P}<0.001)$. Finally, referring to the results of the correlation and partial correlation between the expression of DMBT1 and the expression PDK-1 in the thyroid carcinoma that DMBT1 and PDK-1 were negatively correlated in the thyroid carcinoma $(r=-0.889$, $\mathrm{P}<0.001$ ), this study made a speculation that the pathological features of thyroid carcinoma were closely related to the expression of DMBT1 and PDK-1, with a highly expressed PDK-1 in it to promote the development of thyroid carcinoma, and with a lowly expressed DMBT1 in it to possibly inhibit the thyroid carcinoma. Despite the few reports about the relationship between the expression of DMBT1 and PDK-1 and the clinicopathological features of thyroid carcinoma in patients, some previous reports on the thyroid carcinoma are still helpful to this study. Following the detection of the expression of DMBT1 and PDK-1, the ROC curve was drawn to investigate the diagnostic value of DMBT1 alone, PDK-1 alone and the combination of the two for thyroid carcinoma, leading to the results that the sensitivity and specificity of the combined detection of DMBT1 and PDK-1 for thyroid carcinoma detection were significantly higher than the single detection of DMBT1 or PDK-1. No study of the diagnostic value of DMBT1 and PDK-1 for the thyroid carcinoma has been reported so far, so this study which specifically explored the correlation between the expression of DMBT1 or PDK-1 and the occurrence and development of the thyroid carcinoma, is of certain significance for the diagnosis and clinical treatment of thyroid carcinoma. Finally, with the research results and 
some supportive views by other studies on hand, this study draw the conclusion that monitoring the expression of DMBT1 or PDK-1 in the protein had certain clinical diagnostic and therapeutic significance for the occurrence and development of the thyroid carcinoma, and that the combined detection of the expression changes of DMBT1 and PDK-1 could diagnose the thyroid carcinoma conveniently, quickly, and accurately, worthy of clinical promotion.

Considering the limited resources and the small number of research subjects, contingency was possible in this study. In the future, more time and energy will be invested in the improvement of this research to achieve better study results.

In summary, PDK-1 was highly expressed in the thyroid carcinoma, while the DMBT1 was lowly expressed. The combined detection of DMBT1 and PDK-1 could improve the accuracy of diagnosing the thyroid carcinoma to reduce the rate of misdiagnosis.

\section{Acknowledgements}

Not applicable.

\section{Funding}

No funding was received.

\section{Availability of data and materials}

The datasets used and/or analyzed during the present study are available from the corresponding author on reasonable request.

\section{Authors' contributions}

ZS analyzed and interpreted the general data of patients and drafted this paper. ZS and LX were responsible for ELISA. Both authors read and approved the final manuscript.

\section{Ethics approval and consent to participate}

The study was approved by the Ethics Committee of The Second People's Hospital of Lianyungang (Lianyungang, China). Patients who participated in this research had complete clinical data. The signed informed consents were obtained from the patients or the guardians.

\section{Patient consent for publication}

Not applicable.

\section{Competing interests}

The authors declare that they have no competing interests.

\section{References}

1. Xing M: BRAF mutation in thyroid cancer. Endocr Relat Cancer 12: 245-262, 2005.

2. Jemal A, Bray F, Center MM, Ferlay J, Ward E and Forman D: Global cancer statistics. CA Cancer J Clin 61: 69-90, 2011.

3. Chen W, Zheng R, Baade PD, Zhang S, Zeng H, Bray F, Jemal A, Yu XQ and He J: Cancer statistics in China, 2015. CA Cancer J Clin 66: 115-132, 2016.
4. Jabbour E, Deininger $M$ and Hochhaus A: Management of adverse events associated with tyrosine kinase inhibitors in the treatment of chronic myeloid leukemia. Leukemia 25: 201-210, 2011.

5. Braidotti P, Nuciforo PG, Mollenhauer J, Poustka A, Pellegrini C, Moro A, Bulfamante G, Coggi G, Bosari S and Pietra GG: DMBT1 expression is down-regulated in breast cancer. BMC Cancer 4: 46, 2004.

6. Imai MA, Moriya T, Imai FL, Shiiba M, Bukawa H, Yokoe H, Uzawa K and Tanzawa H: Down-regulation of DMBT1 gene expression in human oral squamous cell carcinoma. Int J Mol Med 15: 585-589, 2005.

7. Hernandez BY, Green MD, Cassel KD, Pobutsky AM, Vu V and Wilkens LR: Preview of Hawaii cancer facts and figures 2010. Hawaii Med J 69: 223-224, 2010.

8. Davies L and Welch HG: Increasing incidence of thyroid cancer in the United States, 1973-2002. JAMA 295: 2164-2167, 2006.

9. Mazzaferri EL and Jhiang SM: Long-term impact of initial surgical and medical therapy on papillary and follicular thyroid cancer. Am J Med 97: 418-428, 1994.

10. Gagliardi PA, Puliafito A and Primo L: PDK1: At the crossroad of cancer signaling pathways. Semin Cancer Biol 48: 27-35, 2018.

11. Leroux AE, Schulze JO and Biondi RM: AGC kinases, mechanisms of regulation and innovative drug development. Semin Cancer Biol 48: 1-17, 2018.

12. Arsenic R: Immunohistochemical analysis of PDK1 expression in breast cancer. Diagn Pathol 9: 82, 2014.

13. Han L, Zhang G, Zhang N, Li H, Liu Y, Fu A and Zheng Y: Prognostic potential of microRNA-138 and its target mRNA PDK1 in sera for patients with non-small cell lung cancer. Med Oncol 31: 129, 2014.

14. Ferro R and Falasca M: Emerging role of the KRAS-PDK1 axis in pancreatic cancer. World J Gastroenterol 20: 10752-10757, 2014.

15. Zheng N, Ding X, Sun A and Jahan R: PDK1 activity regulates proliferation, invasion and growth of hemangiomas. Cell Physiol Biochem 36: 1903-1910, 2015.

16. Lian S, Shao Y, Liu H, He J, Lu W, Zhang Y, Jiang Y and Zhu J: PDK1 induces JunB, EMT, cell migration and invasion in human gallbladder cancer. Oncotarget 6: 29076-29086, 2015.

17. Mollenhauer J, Holmskov U, Wiemann S, Krebs I, Herbertz S, Madsen J, Kioschis P, Coy JF and Poustka A: The genomic structure of the DMBT1 gene: evidence for a region with susceptibility to genomic instability. Oncogene 18: 6233-6240, 1999.

18. Mollenhauer J, Wiemann S, Scheurlen W, Korn B, Hayashi Y, Wilgenbus KK, von Deimling A and Poustka A: DMBT1, a new member of the SRCR superfamily, on chromosome 10q25.3-26.1 is deleted in malignant brain tumours. Nat Genet 17: 32-39, 1997.

19. Smith HA and Kang Y: The metastasis-promoting roles of tumor-associated immune cells. J Mol Med (Berl) 91: 411-429, 2013.

20. Deng H, Gao YB, Wang HF, Jin XL and Xiao JC: Expression of deleted in malignant brain tumours 1 (DMBT1) relates to the proliferation and malignant transformation of hepatic progenitor cells in hepatitis B virus-related liver diseases. Histopathology 60: 249-260, 2012.

21. Du J, Guan M, Fan J and Jiang H: Loss of DMBT1 expression in human prostate cancer and its correlation with clinical progressive features. Urology 77: 509.e9-509.e13, 2011.

22. Zöchbauer-Müller S, Fong KM, Virmani AK, Geradts J, Gazdar AF and Minna JD: Aberrant promoter methylation of multiple genes in non-small cell lung cancers. Cancer Res 61: 249-255, 2001.

23. Mollenhauer J, Herbertz S, Helmke B, Kollender G, Krebs I, Madsen J, Holmskov U, Sorger K, Schmitt L, Wiemann S, et al: Deleted in Malignant Brain Tumors 1 is a versatile mucin-like molecule likely to play a differential role in digestive tract cancer. Cancer Res 61: 8880-8886, 2001.

24. Ridnour LA, Cheng RY, Switzer CH, Heinecke JL, Ambs S, Glynn S, Young HA, Trinchieri G and Wink DA: Molecular pathways: toll-like receptors in the tumor microenvironment-poor prognosis or new therapeutic opportunity. Clin Cancer Res 19: 1340-1346, 2013.

This work is licensed under a Creative Commons Attribution-NonCommercial-NoDerivatives 4.0 International (CC BY-NC-ND 4.0) License. 